\title{
ON A CONJECTURE BY WILF ABOUT THE FROBENIUS NUMBER
}

\author{
ALESSIO MOSCARIELLO AND ALESSIO SAMMARTANO
}

\begin{abstract}
Given coprime positive integers $a_{1}<\cdots<a_{d}$, the Frobenius number $F$ is the largest integer which is not representable as a non-negative integer combination of the $a_{i}$. Let $g$ denote the number of all non-representable positive integers: Wilf conjectured that $d \geq \frac{F+1}{F+1-g}$. We prove that for every fixed value of $\left\lceil\frac{a_{1}}{d}\right\rceil$ the conjecture holds for all values of $a_{1}$ which are sufficiently large and are not divisible by a finite set of primes. We also propose a generalization in the context of one-dimensional local rings and a question on the equality $d=\frac{F+1}{F+1-g}$.
\end{abstract}

\section{INTRODUCTION}

The Diophantine Frobenius problem or coin problem consists of studying the greatest integer $F$, called the Frobenius number, that is not representable as a linear combination of given $d$ coprime positive integers $a_{1}<\cdots<a_{d}$ with coefficients in $\mathbb{N}$. The problem has stimulated much research over the past decades, due to the applications to several areas of pure and applied mathematics including Coding Theory, Linear Algebra, Combinatorics and Commutative Algebra. The monograph [11] explores several viewpoints about the problem.

Let $\mathcal{S}=\left\{\sum_{i=1}^{d} \lambda_{i} a_{i}: \lambda_{i} \in \mathbb{N}\right\}$ be the numerical semigroup generated by the $a_{i}$. The condition $\operatorname{gcd}\left(a_{1}, \ldots, a_{d}\right)=1$ implies that the set of gaps $\mathbb{N} \backslash \mathcal{S}$ is finite and its cardinality $g=|\mathbb{N} \backslash \mathcal{S}|$ is known as the genus of $\mathcal{S}$. In $1978 \mathrm{H}$. S. Wilf proposed a lower bound for the number of generators of $\mathcal{S}$ in terms of the Frobenius number and the genus (cf. [14]):

$$
d \geq \frac{F+1}{F+1-g} .
$$

Although the problem has been considered by several authors (cf. [1], 3], 7], 8], 9], 13, [15]) only special cases have been solved and it remains wide open. The approach we follow in this paper is to study the poset structure of the so-called Apéry set once the value of the ratio $\rho=\left\lceil\frac{a_{1}}{d}\right\rceil$ is fixed. The cases $\rho=1$ and $\rho=2$ were solved in [7] and [13] respectively; in this work we focus on the general case. Our main result is the following:

Theorem 1. For every value of $\rho=\left\lceil\frac{a_{1}}{d}\right\rceil$ Wilf's inequality (1) holds if $a_{1}$ is large enough and the prime factors of $a_{1}$ are greater than or equal to $\rho$.

The explicit lower bound for $a_{1}$ in Theorem 1 is $a_{1} \geq \frac{\rho\left(3 \rho^{2}-\rho-4\right)\left(3 \rho^{2}-\rho-2\right)}{8(\rho-2)}$.

In the first section of the paper we recall some definitions and preliminary results, whereas the second section is devoted to proving Theorem 1. We conclude the paper with a word about the equality in (1) and a discussion of the conjecture in the context of Commutative Algebra.

2010 Mathematics Subject Classification. Primary: 11D07; Secondary: 11B75, $20 \mathrm{M} 14$.

Key words and phrases. Diophantine Frobenius problem; coin problem; Wilf's conjecture; numerical semigroup; Apéry set; length inequality; one-dimensional local ring. 


\section{Preliminaries}

We assume without loss of generality that $a_{1}, \ldots, a_{d}$ are the minimal generators of $\mathcal{S}$, i.e. no proper subset generates the semigroup; this implies that $d \leq a_{1}$. The Apéry set is defined as

$$
\operatorname{Ap}(\mathcal{S})=\left\{\omega \in \mathcal{S}: \omega-a_{1} \notin \mathcal{S}\right\}
$$

and thus it consists of the smallest elements of $\mathcal{S}$ in each residue class modulo $a_{1}$. It follows that $0 \in \operatorname{Ap}(\mathcal{S}),|\operatorname{Ap}(\mathcal{S})|=a_{1}$ and that $F+a_{1}$ is the largest element in $\operatorname{Ap}(\mathcal{S})$. We list the elements of $\operatorname{Ap}(\mathcal{S})$ increasingly setting $\operatorname{Ap}(\mathcal{S})=\left\{\omega_{0}<\omega_{1}<\cdots<\omega_{a_{1}-1}\right\}$, notice that we have $\omega_{0}=0, \omega_{1}=a_{2}, \omega_{a_{1}-1}=F+a_{1}$. We establish a partial order on $\mathbb{N}$ by setting $n_{1} \preceq n_{2}$ if there exists $s \in \mathcal{S}$ such that $n_{1}+s=n_{2}$ and we consider throughout the paper the poset $(\operatorname{Ap}(\mathcal{S}) \backslash\{0\}, \preceq)$. The minimal elements in $(\operatorname{Ap}(\mathcal{S}) \backslash\{0\}, \preceq)$ are exactly $\left\{a_{2}, \ldots, a_{d}\right\}$ and if $\tau \in \mathcal{S}, \omega \in \operatorname{Ap}(\mathcal{S}), \tau \preceq \omega$ then $\tau \in \operatorname{Ap}(\mathcal{S})$. We refer to [12] for details on numerical semigroups.

Now we give further definitions in order to introduce a reformulation of Wilf's inequality. For each $k \in \mathbb{N}$ let $I_{k}=\left[k a_{1},(k+1) a_{1}-1\right]$ and $n_{k}=\left|\mathcal{S} \cap[0, F] \cap I_{k}\right|$. We write

$$
F+1=Q a_{1}+R
$$

with $Q, R \in \mathbb{N}$ and $2 \leq R \leq a_{1}$. Note that $R \neq 1$ as $F \notin \mathcal{S}$ and that $I_{Q}$ is the interval containing the Frobenius number. We define the numbers

$$
\eta_{j}=\left|\left\{k \in \mathbb{N}:\left|I_{k} \cap \mathcal{S}\right|=j\right\}\right| \quad \text { and } \quad \epsilon_{j}=\left|\left\{k \in \mathbb{N}:\left|I_{k} \cap \mathcal{S}\right|=j, 0 \leq k \leq Q-1\right\}\right|
$$

for each $j \in\left\{1,2, \ldots, a_{1}-1\right\}$. In other words, $\eta_{j}$ counts the intervals $I_{k}$ containing exactly $j$ elements of $\mathcal{S}$, while $\epsilon_{j}$ only counts such intervals among the first $Q$. The two definitions differ slightly, and the numbers $\eta_{j}$ can be expressed in terms of $\operatorname{Ap}(\mathcal{S})$ :

Lemma 2 ([13], 12, 13). The following properties hold:

(1) $\epsilon_{j}=\eta_{j}-1$ if $j=\left|I_{Q} \cap \mathcal{S}\right|$ and $\epsilon_{j}=\eta_{j}$ otherwise;

(2) $\eta_{j}=\left\lfloor\frac{\omega_{j}}{a_{1}}\right\rfloor-\left\lfloor\frac{\omega_{j-1}}{a_{1}}\right\rfloor$ for all $1 \leq j \leq a_{1}-1$.

The numbers $\epsilon_{j}$ give rise to an equivalent formulation of (1):

Proposition 3 ([13], 10, 11). We have the equation

$$
d(F+1-g)-(F+1)=\sum_{j=1}^{a_{1}-1} \epsilon_{j}\left(j d-a_{1}\right)+\left(n_{Q} d-R\right)=: \Delta .
$$

\section{Proof of THE MAIN THEOREM}

In this section we prove Theorem 1 using Proposition 3, We need a series of lemmas first.

Lemma 4. If $x \in \mathbb{N} \backslash\{0\}$ is smaller than every prime factor of $a_{1}$ then $\left\lfloor\frac{\omega_{x}}{a_{1}}\right\rfloor \leq x\left\lfloor\frac{\omega_{1}}{a_{1}}\right\rfloor+x-1$. Proof. The assumption on $x$ implies that $i \omega_{1} \not \equiv j \omega_{1}\left(\bmod a_{1}\right)$ for any $0 \leq i<j \leq x$ : in fact $i \omega_{1} \equiv j \omega_{1}\left(\bmod a_{1}\right)$ yields the contradiction $(j-i) \omega_{1} \equiv 0\left(\bmod a_{1}\right)$ as $\operatorname{gcd}\left(j-i, a_{1}\right)=1$ and $\omega_{1} \not \equiv 0\left(\bmod a_{1}\right)$. Thus the subset $\left\{\omega_{1}, 2 \omega_{1}, \ldots, x \omega_{1}\right\} \subseteq \mathcal{S}$ covers $x$ different residue classes modulo $a_{1}$, hence there are elements of at least $x$ different classes less than or equal to $x \omega_{1}$ in $\mathcal{S}$. By definition of Apéry set we deduce that $\omega_{x} \leq x \omega_{1}$ and $\left\lfloor\frac{\omega_{x}}{a_{1}}\right\rfloor \leq\left\lfloor\frac{x \omega_{1}}{a_{1}}\right\rfloor \leq x\left\lfloor\frac{\omega_{1}}{a_{1}}\right\rfloor+x-1$.

Lemma 5. If $y \in \mathbb{N}$ satisfies $y \geq 2$ and $a_{1}-d \geq\left(\begin{array}{l}y \\ 2\end{array}\right)+1$ then $\omega_{a_{1}-1} \geq \omega_{y}+\omega_{1}$. In particular, $\left\lfloor\frac{\omega_{a_{1}-1}}{a_{1}}\right\rfloor \geq\left\lfloor\frac{\omega_{y}}{a_{1}}\right\rfloor+\left\lfloor\frac{\omega_{1}}{a_{1}}\right\rfloor$ and $F>\omega_{y}$. 
Proof. Since the minimal elements in $(\operatorname{Ap}(\mathcal{S}) \backslash\{0\}, \preceq)$ are exactly $\left\{a_{2}, \ldots, a_{d}\right\}$ and $|\operatorname{Ap}(\mathcal{S})|=a_{1}$, there are at least $\left(\begin{array}{l}y \\ 2\end{array}\right)+1$ non-minimal elements in $(\operatorname{Ap}(\mathcal{S}) \backslash\{0\}, \preceq)$. The set $\mathcal{Y}=\left\{\omega_{i}+\omega_{j} \mid 1 \leq i \leq\right.$ $j \leq y-1\}$ contains at most $\left(\begin{array}{l}y \\ 2\end{array}\right)$ distinct elements, so there exists $\tau \in \operatorname{Ap}(\mathcal{S}) \backslash\left(\left\{0, a_{2}, \ldots, a_{d}\right\} \cup \mathcal{Y}\right)$. In particular $\tau$ is not minimal, thus $\tau=\omega_{h}+\omega_{k}$ for some $1 \leq h \leq k$, and $\tau \notin \mathcal{Y}$ yields $k \geq y$. Finally we have $\omega_{a_{1}-1} \geq \tau=\omega_{h}+\omega_{k} \geq \omega_{1}+\omega_{y}$. The inequality with the floor function follows immediately, and from $\omega_{1}>a_{1}$ we obtain $\omega_{a_{1}-1}=F+a_{1} \geq \omega_{y}+\omega_{1}>\omega_{y}+a_{1}$ and $F>\omega_{y}$.

Lemma 6. If $y, z \in \mathbb{N}$ satisfy $y \geq 2, y \geq z, a_{1}-d \geq\left(\begin{array}{l}y \\ 2\end{array}\right)+1$, and $\left\lfloor\frac{\omega_{a_{1}-1}}{a_{1}}\right\rfloor=\left\lfloor\frac{\omega_{z}}{a_{1}}\right\rfloor+\left\lfloor\frac{\omega_{1}}{a_{1}}\right\rfloor$ then $n_{Q} \geq y-z+3$.

Proof. Fix $z \leq i \leq y$, by Lemma 5 we have that $F+a_{1} \geq \omega_{1}+\omega_{y} \geq \omega_{1}+\omega_{i}$ and

$$
\left\lfloor\frac{\omega_{a_{1}-1}}{a_{1}}\right\rfloor \geq\left\lfloor\frac{\omega_{y}}{a_{1}}\right\rfloor+\left\lfloor\frac{\omega_{1}}{a_{1}}\right\rfloor \geq\left\lfloor\frac{\omega_{i}}{a_{1}}\right\rfloor+\left\lfloor\frac{\omega_{1}}{a_{1}}\right\rfloor \geq\left\lfloor\frac{\omega_{z}}{a_{1}}\right\rfloor+\left\lfloor\frac{\omega_{1}}{a_{1}}\right\rfloor=\left\lfloor\frac{\omega_{a_{1}-1}}{a_{1}}\right\rfloor
$$

and this leads to $\left\lfloor\frac{\omega_{y}}{a_{1}}\right\rfloor=\left\lfloor\frac{\omega_{i}}{a_{1}}\right\rfloor=\left\lfloor\frac{\omega_{z}}{a_{1}}\right\rfloor$. From $F+1=Q a_{1}+R$ we obtain $(Q+1) a_{1}+(R-1) \geq$ $\omega_{1}+\omega_{i}$. Dividing by $a_{1}$ we get $\omega_{1}=q_{1} a_{1}+r_{1}$ and $\omega_{i}=q_{i} a_{1}+r_{i}$ with $0 \leq r_{i} \leq a_{1}-1$ and it follows that $Q+1=\left\lfloor\frac{\omega_{a_{1}-1}}{a_{1}}\right\rfloor=\left\lfloor\frac{\omega_{z}}{a_{1}}\right\rfloor+\left\lfloor\frac{\omega_{1}}{a_{1}}\right\rfloor=\left\lfloor\frac{\omega_{i}}{a_{1}}\right\rfloor+\left\lfloor\frac{\omega_{1}}{a_{1}}\right\rfloor=q_{1}+q_{i}$. From $\omega_{a_{1}-1} \geq \omega_{i}+\omega_{1}$ we obtain $(Q+1) a_{1}+(R-1) \geq\left(q_{i}+q_{1}\right) a_{1}+\left(r_{i}+r_{1}\right)=(Q+1) a_{1}+\left(r_{i}+r_{1}\right)$, thus $R-1 \geq r_{i}+r_{1}$. In particular, $r_{1}, r_{i}<R$ : we conclude that the elements $Q a_{1}, \omega_{1}+l_{1} a_{1}$ and $\omega_{i}+l_{i} a_{1}$ belong to $\mathcal{S} \cap I_{Q} \cap[0, F]$ for suitable $l_{1}, l_{i} \in \mathbb{N}$ for each $z \leq i \leq y$ so that $n_{Q} \geq 3+y-z$.

Lemma 7. If $y \in \mathbb{N}$ satisfies $y \geq 2$ and $a_{1}-d \geq\left(\begin{array}{l}y \\ 2\end{array}\right)+1$ then $n_{Q-1} \geq y+2-n_{Q}$.

Proof. By Lemma 5 we have $a_{1}<\omega_{y}<F$, whence $Q>0$ and $\left\{\omega_{0}, \ldots, \omega_{y}\right\} \subseteq[0, F]$. At most $n_{Q}-1$ of these elements belong to $\mathcal{S} \cap I_{Q} \cap[0, F]$, because $Q a_{1} \notin \operatorname{Ap}(\mathcal{S})$. Hence there are at least $y+2-n_{Q}$ elements $\omega_{i}$ smaller than $Q a_{1}$, that is, $\omega_{0}<\cdots<\omega_{y-n_{Q}+1}<Q a_{1}$ : we conclude that $\omega_{i}+l_{i} a_{1} \in I_{Q-1}$ for suitable $l_{i} \in \mathbb{N}$ for each $0 \leq i \leq y-n_{Q}+1$ so $n_{Q-1} \geq y+2-n_{Q}$.

We are now ready to prove the main result.

Proof of Theorem 1. Let $\rho=\left\lceil\frac{a_{1}}{d}\right\rceil$. Since the cases $\rho=1,2$ have been solved, we assume $\rho \geq 3$. Consider the integers

$$
y=\frac{3 \rho^{2}-\rho-4}{2} \quad \text { and } \quad z=\frac{\rho^{2}+\rho-2}{2}
$$

notice that $y \geq z \geq \rho+2$. We are going to make the assumption that $a_{1} \geq \frac{\rho}{\rho-2}\left(\begin{array}{l}y \\ 2\end{array}\right)$, so that the condition appearing in Lemmas 5, 6, 7 is satisfied:

$$
a_{1}-d \geq(\rho-1) d+1-d=(\rho-2) d+1 \geq \frac{\rho-2}{\rho} a_{1}+1 \geq\left(\begin{array}{l}
y \\
2
\end{array}\right)+1 .
$$

Our task is to prove (1) by showing that the quantity $\Delta$ of Proposition 3 is non-negative; we will actually show that $\Delta>0$. We are going to break the summation in two parts, at the index $j=\rho$. For the first part we use both properties of Lemma 2 and obtain:

$$
\begin{aligned}
\sum_{j=1}^{\rho} \epsilon_{j}\left(j d-a_{1}\right) & \geq \sum_{j=1}^{\rho} \eta_{j}\left(j d-a_{1}\right)-\left(\rho d-a_{1}\right)=\sum_{j=1}^{\rho}\left(\left\lfloor\frac{\omega_{j}}{a_{1}}\right\rfloor-\left\lfloor\frac{\omega_{j-1}}{a_{1}}\right\rfloor\right)\left(j d-a_{1}\right)-\left(\rho d-a_{1}\right) \\
& =\left\lfloor\frac{\omega_{\rho}}{a_{1}}\right\rfloor\left(\rho d-a_{1}\right)-d \sum_{j=1}^{\rho-1}\left\lfloor\frac{\omega_{j}}{a_{1}}\right\rfloor-\left(\rho d-a_{1}\right) \geq-d \sum_{j=1}^{\rho-1}\left\lfloor\frac{\omega_{j}}{a_{1}}\right\rfloor
\end{aligned}
$$


where we used that $\omega_{0}=0$ and $\omega_{\rho}>\omega_{1}>a_{1}$. By the assumption on the factors of $a_{1}$ we can use Lemma 4 for each $j<\rho$, yielding

$$
\sum_{j=1}^{\rho-1}\left\lfloor\frac{\omega_{j}}{a_{1}}\right\rfloor \leq \sum_{j=1}^{\rho-1}\left(j\left\lfloor\frac{\omega_{1}}{a_{1}}\right\rfloor+j-1\right)=\left(\begin{array}{l}
\rho \\
2
\end{array}\right)\left\lfloor\frac{\omega_{1}}{a_{1}}\right\rfloor+\left(\begin{array}{c}
\rho-1 \\
2
\end{array}\right)
$$

and therefore

$$
\sum_{j=1}^{\rho} \epsilon_{j}\left(j d-a_{1}\right) \geq-\frac{d\left(\rho^{2}-\rho\right)}{2}\left\lfloor\frac{\omega_{1}}{a_{1}}\right\rfloor-\frac{d\left(\rho^{2}-3 \rho+2\right)}{2} .
$$

Moreover, since $\frac{1}{2} d\left(\rho^{2}-\rho\right) \leq \frac{1}{2} d\left(\rho^{2}-\rho\right)+\rho d-a_{1}=\frac{1}{2} d\left(\rho^{2}+\rho\right)-a_{1}=(z+1) d-a_{1}$ then

$$
\sum_{j=1}^{\rho} \epsilon_{j}\left(j d-a_{1}\right) \geq-\left((z+1) d-a_{1}\right)\left\lfloor\frac{\omega_{1}}{a_{1}}\right\rfloor-\frac{d\left(\rho^{2}-3 \rho+2\right)}{2} .
$$

For the second part of the summation in $\Delta$, as $z+1 \geq \rho+3$ we can write

$$
\begin{gathered}
\sum_{j=\rho+1}^{a_{1}-1} \epsilon_{j}\left(j d-a_{1}\right) \geq \sum_{j=z+1}^{a_{1}-1} \epsilon_{j}\left(j d-a_{1}\right) \geq \sum_{j=z+1}^{a_{1}-1} \epsilon_{j}\left((z+1) d-a_{1}\right)=\left((z+1) d-a_{1}\right) \sum_{j=z+1}^{a_{1}-1} \epsilon_{j} \geq \\
\geq\left((z+1) d-a_{1}\right)\left(\sum_{j=z+1}^{a_{1}-1} \eta_{j}-1\right)=\left((z+1) d-a_{1}\right)\left(\left\lfloor\frac{\omega_{a_{1}-1}}{a_{1}}\right\rfloor-\left\lfloor\frac{\omega_{z}}{a_{1}}\right\rfloor-1\right)
\end{gathered}
$$

where we used again both properties of Lemma 2 in the last inequality. Combining the two parts gives

$$
\begin{gathered}
\Delta=\sum_{j=1}^{a_{1}-1} \epsilon_{j}\left(j d-a_{1}\right)+\left(n_{Q} d-R\right) \geq \\
\left(\left\lfloor\frac{\omega_{a_{1}-1}}{a_{1}}\right\rfloor-\left\lfloor\frac{\omega_{z}}{a_{1}}\right\rfloor-\left\lfloor\frac{\omega_{1}}{a_{1}}\right\rfloor-1\right)\left((z+1) d-a_{1}\right)-\frac{d\left(\rho^{2}-3 \rho+2\right)}{2}+\left(n_{Q} d-R\right)=: \Pi .
\end{gathered}
$$

Since $z \leq y$, by Lemma 5 we have the inequality $\left\lfloor\frac{\omega_{a_{1}-1}}{a_{1}}\right\rfloor \geq\left\lfloor\frac{\omega_{z}}{a_{1}}\right\rfloor+\left\lfloor\frac{\omega_{1}}{a_{1}}\right\rfloor$. If the equality holds, we can use the bound for $n_{Q}$ in Lemma 6 obtaining

$$
\begin{aligned}
\Delta & \geq \Pi \geq-\left((z+1) d-a_{1}\right)-\frac{d\left(\rho^{2}-3 \rho+2\right)}{2}+((y-z+3) d-R)= \\
& =d\left(y-2 z-\frac{\rho^{2}-3 \rho+2}{2}+2\right)+\left(a_{1}-R\right)=d+\left(a_{1}-R\right)>0
\end{aligned}
$$

by the definitions of $y$ and $z$ and the fact that $a_{1} \geq R$. Suppose now we have the strict inequality $\left\lfloor\frac{\omega_{a_{1}-1}}{a_{1}}\right\rfloor>\left\lfloor\frac{\omega_{z}}{a_{1}}\right\rfloor+\left\lfloor\frac{\omega_{1}}{a_{1}}\right\rfloor$. In this case the first piece in $\Pi$ is non-negative because $(z+1) d-a_{1} \geq \rho d-a_{1} \geq 0$, thus we can ignore it:

$$
\Pi \geq-\frac{d\left(\rho^{2}-3 \rho+2\right)}{2}+\left(n_{Q} d-R\right)
$$

If $n_{Q} \geq \frac{1}{2}\left(\rho^{2}-\rho+4\right)$, then $\Delta \geq \Pi \geq \rho d-R+d \geq \rho d-a_{1}+d>0$. Suppose finally that $n_{Q} \leq \frac{1}{2}\left(\rho^{2}-\rho+2\right)$. By Lemma 7 we know that $n_{Q-1} \geq y+2-n_{Q}$, i.e. $\mathcal{S} \cap I_{Q-1}$ contains at least $y+2-n_{Q}$ elements, and in this case from $\rho \geq 3$ it follows

$$
y+2-n_{Q} \geq \frac{3 \rho^{2}-\rho-4}{2}+2-\frac{\rho^{2}-\rho+2}{2}=\rho^{2}-1 \geq \frac{\rho^{2}}{2}+\frac{3 \rho}{2}-1 \geq \frac{\rho^{2}+\rho-2}{2}+\rho \geq z+3 .
$$


Let $j_{0}=n_{Q-1} \geq y+2-n_{Q} \geq z+3 \geq \rho+1$, we have $\epsilon_{j_{0}} \geq 1$ and therefore

$$
\sum_{j=\rho+1}^{a_{1}-1} \epsilon_{j}\left(j d-a_{1}\right) \geq \sum_{j=z+1}^{a_{1}-1} \epsilon_{j}\left(j d-a_{1}\right) \geq\left(j_{0} d-a_{1}\right)-\left((z+1) d-a_{1}\right)+\sum_{z+1}^{a_{1}-1} \epsilon_{j}\left((z+1) d-a_{1}\right)
$$

it follows that

$$
\begin{gathered}
\Delta=\sum_{j=1}^{a_{1}-1} \epsilon_{j}\left(j d-a_{1}\right)+\left(n_{Q} d-R\right) \geq \Pi+\left(y+2-n_{Q}-z-1\right) d \geq \\
-\frac{d\left(\rho^{2}-3 \rho+2\right)}{2}-R+(y-z+1) d=\frac{d\left(\rho^{2}+\rho-2\right)}{2}-R \geq \frac{d(3 \rho+3-2)}{2}-R \geq(\rho d-R)+\frac{1}{2}>0
\end{gathered}
$$

where we used that $\rho \geq 3$ and $\rho d \geq a_{1} \geq R$.

We have shown that $\Delta>0$ in each case and the theorem is thus proved.

\section{TWO FURTHER PROBLEMS}

Whenever we have a bound, it is natural to ask ourselves whether the bound is sharp and, in case it is, to try to characterize the instances in which the bound is achieved. In his original paper [14, Wilf also asked whether the equality in (11) is attained if and only if $d=a_{1}$ and $a_{i}=a_{1}+(i-1)$ for $i=2, \ldots, a_{1}$. This is not the case: a simple counterexample is $a_{1}=3, a_{2}=5$. However, we believe that equality can only occur in two cases.

Question 8. Is it true that the equality $F+1=d(F+1-g)$ holds if and only if either $d=2$ or $d=a_{1}$ and there exists $K \in \mathbb{N}$ such that $a_{i}=K a_{1}+(i-1)$ for $i=2, \ldots, a_{1}$ ?

Note that in the latter case the numerical semigroup has the form

$$
\mathcal{S}=\left\{0, a_{1}, 2 a_{1}, \ldots,(K-1) a_{1}, K a_{1}, K a_{1}+1, K a_{1}+2, \ldots\right\}
$$

and the equality follows from Proposition 3 , while in the former case the equality was already known to Sylvester (cf. [12, 2.12]), so the interesting part is the "only if". We observe that, in order to show that these are the only two cases, it suffices to prove that either $d=2$ or $d=a_{1}$. In fact, if $d=a_{1}$ and $F+1=d(F+1-g)$ then Proposition 3 implies the equation

$$
\Delta=\sum_{j=1}^{a_{1}-1} \epsilon_{j}(j-1) a_{1}+\left(n_{Q} a_{1}-R\right)=0
$$

and since $n_{Q} \geq 1, a_{1} \geq R$ we conclude that $\epsilon_{j}=0$ for every $j \geq 2, n_{Q}=1, R=a_{1}$; it follows that $a_{i}=(Q+1) a_{1}+(i-1)$ for $i=2, \ldots, a_{1}$.

We have verified via GAP (cf. [6]) that Question 8 has an affirmative answer when $g \leq 35$. Further evidence in its favor is given by the proof of Theorem 1, as in all the cases investigated therein (where $\rho \geq 3$, hence $d<a_{1}$ ) the strict inequality was actually seen to hold: in other words, we give a positive answer under the assumptions of our theorem.

Finally, we remark that the conjecture has an interpretation in Commutative Algebra in terms of length inequalities. Let $\mathcal{R}=\mathbb{k}\left[\left[t^{a_{1}}, \ldots, t^{a_{d}}\right]\right]$ be the local ring of a monomial curve, where $\mathbb{k}$ is a field. Let $\overline{\mathcal{R}}=\mathbb{k}[[t]]$ be the integral closure of $\mathcal{R}$ in its field of fractions $\mathcal{Q}=\mathbb{k}((t))$ and $\mathfrak{C}=(\mathcal{R}: \mathcal{Q} \overline{\mathcal{R}})$ be the conductor of $\mathcal{R}$ in $\overline{\mathcal{R}}$, that is the largest common ideal of $\mathcal{R}$ and $\overline{\mathcal{R}}$. Denoting by $\ell(\cdot)$ the length of an $\mathcal{R}$-module, the values of $\ell(\overline{\mathcal{R}} / \mathcal{R})$ and $\ell(\mathcal{R} / \mathfrak{C})$ are both measures of the singularity of $\mathcal{R}$ and they are related by $\ell(\mathcal{R} / \mathfrak{C}) \leq \ell(\overline{\mathcal{R}} / \mathcal{R})$, with equality holding if and only if $\mathcal{R}$ is Gorenstein. Under the notation of this paper, the embedding 
dimension of $\mathcal{R}$ is $\operatorname{edim}(\mathcal{R})=d$, whereas $\ell(\overline{\mathcal{R}} / \mathfrak{C})=F+1$ and $\ell(\mathcal{R} / \mathfrak{C})=F+1-g$ (cf. [2, II.1]). Therefore (11) is equivalent to

$$
\ell(\overline{\mathcal{R}} / \mathfrak{C}) \leq \operatorname{edim}(\mathcal{R}) \ell(\mathcal{R} / \mathfrak{C}) .
$$

A similar well-known inequality holds in a more general context: if $\mathcal{R}$ is a one-dimensional Cohen-Macaulay local ring with type $t(\mathcal{R})$ and such that the integral closure $\overline{\mathcal{R}}$ of $\mathcal{R}$ in its total ring of fractions is a finite $\mathcal{R}$-module, then

$$
\ell(\overline{\mathcal{R}} / \mathfrak{C}) \leq(t(\mathcal{R})+1) \ell(\mathcal{R} / \mathfrak{C})
$$

see e.g. [4], [5], 10]. It follows that (2) is satisfied if $t(\mathcal{R})<\operatorname{edim}(\mathcal{R})$, but the two invariants are unrelated in general: J. Backelin exhibited a family of $\operatorname{rings}$ with edim $(\mathcal{R})=4$ and arbitrarily large type (cf. [8]). It would be natural to explore the form (2) of Wilf's inequality for more general classes of rings, therefore we conclude the paper with the following general question.

Question 9. Let $\mathcal{R}$ be a one-dimensional Cohen-Macaulay local ring such that the integral closure of $\mathcal{R}$ in its total ring of fractions is a finite $\mathcal{R}$-module. Under what assumptions do we have $\ell(\overline{\mathcal{R}} / \mathfrak{C}) \leq \operatorname{edim}(\mathcal{R}) \ell(\mathcal{R} / \mathfrak{C})$ ?

\section{REFERENCES}

1. V. Barucci, On propinquity of numerical semigroups and one-dimensional local Cohen Macaulay rings, Commutative Algebra and Applications, pp. 49-60 (2009).

2. V. Barucci, D. E. Dobbs, M. Fontana, Maximality properties in numerical semigroups and applications to one-dimensional analytically irreducible local domains, No. 598. AMS Bookstore (1997).

3. M. Bras-Amors, Fibonacci-like behavior of the number of numerical semigroups of a given genus, Semigroup Forum 76, pp. 379-384 (2008).

4. W. C. Brown, J. Herzog, One dimensional local rings of maximal and almost maximal length, Journal of Algebra 151, pp. 332-347 (1992).

5. D. Delfino, On the inequality $\lambda(\bar{R} / R) \leq t(R) \lambda(R / C)$ for one-dimensional local rings, Journal of Algebra 169, pp. 332-342 (1994).

6. M. Delgado, P. A. García-Sanchez, J. Morais, Numericalsgps, a GAP package for numerical semigroups, Version 0.98 (2013).

7. D. E. Dobbs, G. L. Matthews, On a question of Wilf concerning numerical semigroups, International Journal of Commutative Rings 3 No. 2 (2003).

8. R. Fröberg, C. Gottlieb, R. Häggkvist, On numerical semigroups, Semigroup Forum 35, pp. 63-83 (1986).

9. N. Kaplan, Counting numerical semigroups by genus and some cases of a question of Wilf, Journal of Pure and Applied Algebra 216, pp. 1016-1032 (2012).

10. T. Matsuoka, On the degree of singularity of one-dimensional analytically irreducible noetherian local rings, Kyoto Journal of Mathematics 11, pp. 485-494 (1971).

11. J. L. Ramírez Alfonsín, The Diophantine Frobenius Problem, Oxford University Press (2005).

12. J. C. Rosales, P. A. García Sanchez, Numerical Semigroups, Springer (2009).

13. A. Sammartano, Numerical semigroups with large embedding dimension satisfy Wilfs conjecture, Semigroup Forum 85 , pp. 439-447 (2012).

14. H. S. Wilf, A circle-of-lights algorithm for the money-changing problem, The American Mathematical Monthly 85, pp. 562-565 (1978).

15. A. Zhai, An asymptotic result concerning a question of Wilf, arXiv:1111.2779 (2011).

(Alessio Moscariello) Dipartimento di Matematica e Informatica, Università di Catania, Viale Andrea Doria 6, 95125 CATAnia, Italy

E-mail address: alessio.moscariello@studium.unict.it

(Alessio Sammartano) Department of Mathematics, Purdue University, 150 N. University Street, WEST LAFAYETTE, IN 47907, USA

E-mail address: asammart@math.purdue.edu 